\title{
Educación y tecnología. Un reto hacia el desarrollo humano. La pregunta por la globalización
}

\author{
Dr. Napoleón Murcia Peña ${ }^{1}$
}

\begin{abstract}
Resumen
Partimos de considerar que la globalización es un movimiento histórico - social que va ligado a la movilidad de todos los ambientes y escenarios de la vida del ser humano.

Por eso, la globalización no es una opción, es por el contrario una realidad que se ha venido construyendo desde los comienzos mismos de la historia civilizatoria y que está relacionada con la necesidad humana de la socialización, con la necesidad de descubrir, crear y comunicar.

Palabras clave: Sociedad, globalización, educación, tecnología, cambio, desarrollo.
\end{abstract}

\section{Education and technology \\ A challenge towards the human development. The question by the globalización.}

\begin{abstract}
We start off to consider that the globalization is an historical movement - social that goes bound to the mobility of all the atmospheres and scenes of the life of the human being.

For that reason, the globalization is not an option, is on the contrary a reality that has come constructing from the same beginnings of history civilization and that is related to the human necessity of the socialization, with the necessity to discover, to create and to communicate.

Key words: Society, globalization, education, technology, change, development.
\end{abstract}

1 Decano Facultad de Educación, Universidad de Caldas. Dr. en Ciencias Sociales, Niñez y Juventud. 
La historia está plagada de ejemplos donde se buscaron expandir fronteras territoriales que por muchos medios fueron diseminando los conocimientos y que sirvieron como base para construir otros en latitudes diversas, que es el fundamento real de la globalización.

Por ejemplo, los inventos de la antigua india, como el papel, la imprenta, la pólvora la suspensión de puentes con cadenas de acero, la brújula magnética, la rueda y el molino, fueron conocidos en el mundo occidental gracias al movimiento de globalización que se ha dado en todos los tiempos de la historia, de igual forma, las matemáticas Árabes fueron reconociéndose en los países del oriente.

En esta consideración están de acuerdo autores, como Zygmunt Bauman (1999) y Amartya Sen (2003), entre otros.

Es de considerar, sin embargo que en la actualidad, este movimiento ocurre con gran fuerza, a tal punto que, quien no se deje pernear por esta se condena a la marginalidad no sólo cultural sino, social, política, económica y científico - tecnológica, que implicaría una gran brecha histórica. En el movimiento de globalización, como dice Bauman (p.11) “(...) los efectos de la nueva condición son desiguales, algunos nos volvemos globales, otros quedan detenidos en la localidad, pero ser local en un mundo globalizado es señal de penuria y degradación."

En realidad, la globalización no trae dentro de sí problemas, pues como movimiento histórico social es instituida por el ser humano quien le da la forma y la orienta hacia los senderos que él mismo considera. Los problemas, justamente se originan en el sentido que los detentores del poder económico y político, le están dando al reto de la globalización, pues se aprovechan de las nuevas formas temporales y espaciales creadas por los mass-media, para hacer de esta una forma mas de desplazamiento, manipulación y exclusión.

Esta problemática, es planteada por Amartya Sen, desde la inequidad a partir de las múltiples desigualdades, disparidades en el bienestar, las asimetrías y desequilibrios de poder y oportunidades sociales y económicas que puede generar la globalización, por Bauman desde la construcción de nuevas formas de exclusión, por Sitglitz (2001), a partir de la manipulación e inequidad de la información y Feixa(2000), desde las diferentes formas de exclusión de la generación A, la generación de las redes.

No basta entender que los pobres en todo el mundo requieren de la globalización tanto como los ricos, dice Sen; también es preciso asegurar que obtengan de ella lo que necesitan, por tanto, para abogar por la globalización se requieren reformas institucionales masivas.

Bauman considera que la globalización ha generado nuevas formas en todos los escenarios de la vida del ser humano; nuevos centros de producción de significados y valores, los cuales son extraterritoriales, pues están emancipados de las restricciones locales.

Esto ha traído nuevas distinciones entre ricos y pobres, nomadismo y sedentarismo, normal y anormal, legal a ilegal, exclusión e inclusión y poder.

Al cambiar las formas de relación temporales y espaciales los cuerpos tienen otro significado, no son indispensables, por lo menos en presencia física real, en las comunicaciones ni transacciones, pues la presencia física ya no se requiere mas que como imagen.

Este nuevo concepto de libertad de movimiento virtual ha cambiado las relaciones no sólo comerciales sino del cuerpo Inter e intra subjetivo. Pues al cambiar 


\section{Plumilla Educativan}

las relaciones cara a cara el contacto se remueve a otras esferas mas virtuales, no directas, "Se impuso un espacio cibernético al mundo humano". (caso de los combates)

La comunicación se expresa en una nueva incorporeidad donde como dice Bauman(p.26) "Ios cuerpos no tienen influencia, pero el ciberespacio si la tiene sobre los cuerpos".

En la globalización las condiciones para la motricidad entonces, no son del todo claras, pues el concepto hegemónico de cuerpo físico ha cambiado y ese cambio es un proceso histórico que ha venido menguando la "actividad motriz"de forma aceleradamente progresiva.

\section{Los imaginarios de un cuerpo instrumentalizado}

Las formas de subsistencia del hombre primitivo estaban fundamentadas en la motricidad; la presencia física era la base de la organización social y satisfacción de las necesidades de todo tipo, pero con la invención de los elementos de prolongación del brazo, como la lanza y la flecha, la utilización del cuerpo motricio fue cambiando hacia la atención de acciones manuales antes que motricias globales, devino luego el sedentarismo que implicó nuevos cambios en las funciones corporales y la territorialización del cuerpo. Se construyó un imaginario de cuerpo fijado a la tierra, de cuerpo limitado, de un cuerpo aún activo y presente pero ubicado en un territorio, en un espacio específico; a tal punto que el cuerpo errante era considerado como castigo.

Con la construcción de ese imaginario de cuerpo sedentario, se prepara el feudalismo, en el cual el cual el concepto de cuerpo - motricidad tiene un cambio radical. El cuerpo motricidad, el cuerpo andanza, trabajo, labor, es entonces re- servado para los esclavos y el no trabajo, la no actividad motriz es asumida como privilegio para los detentores del poder.

La corporeidad va perdiendo vitalidad en los nuevos imaginarios y el trabajo corporal como expresión de sentido y subsistencia que antes era la base de la vida, se convierte en un problema de segregación, exclusión e ignorancia. Las religiones que ubican los dioses incorpóreos son muestra de ese cambio, a tal punto que el cuerpo es asumido como la desgracia del espíritu para lograr trascender hacia el don de la sacralizad. Existe sin embargo una gran paradoja en este imaginario religioso, pues pese a que lo corporal es obstáculo y pecado, lo motricio es expresión de gratitud, sentimiento y alegría, lo cual es expresado en fiestas y rituales religiosos.

Con el imperio de la razón, el imaginario sobre la trascendencia no cambia mucho, pues tan sólo es otro el sentido de lo trascendental, ya lo espiritual no sería la síntesis sino la razón, Reinaldo Suárez(1991) analiza esta influencia desde el idealismo, considerando que existían discriminaciones entre los artesanos y los eruditos de la época.

Posteriormente sería la sociedad industrial la que vuelve a revitalizar el imaginario motricio dándole un sentido de producción y consumo. Sin embargo es de considerar que ese imaginario "revitalizado" no compartía los mismos criterios de generalidad, vida y subsistencia, del imaginario motricio primitivo, pues en los nuevos imaginarios construidos se sigue manteniendo el privilegio del no movimiento, de una especie de "lúdica del expectante" en la cual los obreros son considerados como fábricas, como mano de obra, y los gustos del no trabajo físico y la asistencia a eventos que impliquen pasividad y quietud son asumidos como el ideal de descanso. Las nuevas generaciones buscan no ser obreros, como 
sus padres, y ocupar puestos que no los intrumentalicen del todo.

Justamente, el concepto de cuerpo instrumental, de cuerpo función surge con gran fuerza en esta sociedad; la motricidad considerada como expresión de vida es cambiada por el rendimiento motriz al ser asociado con la máquina. "Homo faber", diría Suárez, en el cual el imaginario se vuelca hacia el rendimiento, la productividad y la eficiencia. Aparecen los conceptos de eficiencia y eficacia asociados desde la producción a todas las actividades humanas, dentro de ellas a la motricidad y la corporalidad.

En la era industrial el concepto de corporeidad es relegado a lo especulativo, a lo banal, a lo no positivo, a lo no productivo. El imaginario de cuerpo se reduce a lo corporal y la motricidad humana se reduce a lo motriz. En este marco aparece la educación física, la cual es absorbida por el imaginario hegemónico y reducida al rendimiento y productividad.

Sin embargo la territorialidad seguía siendo la base de las acciones e interacciones sociales, políticas, culturales, científicas, religiosas, éticas, estéticas, expresivas y económicas, incluso en la era industrial; donde a decir de Bauman (p.16), la base del capital estaba en el territorio, estaba ligada al espacio, pues las empresas deberían estar ubicadas en lugares específicos donde acudían los trabajadores.

Pero la sociedad industrial traía dentro de sí el consumo, su hijo mutante que necesariamente debería desarrollarse una vez madura la producción. El consumo entonces genera un nuevo imaginario de instrumentalización corporal, en el cual el cuerpo estaría dotado de capacidades estéticas capaces de provocar y consumir. Capacidades que no son mas que eso, capacidades: para generar modelos y provocar consumos, y no cualidades, no posibilidades de expresión de sentido, no opciones de expresión de corporeidad. Las acciones e interacciones corporales se van reduciendo aún mas, dados las grandes avances en la industria y la comunicación.

En esta nueva sociedad, los conceptos toman nuevas direcciones, los deseos, las necesidades, las posibilidades y los mundos simbólicos se tornan diferentes; los imaginarios se formulan en procura de complacer necesites creadas por los massmedia y la industria cultural, la cual se preocupa por estar creando día a día nuevas necesidades, en lo que radica su éxito y expansión.

Comienza a surgir una nueva sociedad, la sociedad de la información, de la comunicación, la sociedad de los medios masivos y con ella, la necesidad de maximizar la globalización. En esta sociedad las sensibilidades cambian hacia formas digitales y se deja de lado la relación directa con experiencia fenomenológica de lo físico, los tiempos y espacios, se vuelven simultáneos y reducidos y los lenguajes se orientan hacia síntesis fraccionadas con gran fundamento en las imágenes discon tinuas.(Barbero,2003, Lyotard 1998).

\section{Las tecnologías de la información}

En este escenario de la globalidad, las tecnologías de la información aparecen con gran potencia para viabilizar los procesos comunicativos en ambientes de incorporeidad e interritorialidad, lo cual facilita la expansión de la globalidad.

En realidad las tecnologías de la información y las comunicaciones son el gramado que han acogido las sociedades para globalizar su cultura y que utilizan con gran propiedad las nuevas expresiones del capitalismo para impostar sus propósitos. Esto, dado que las tecnologías, son 
el conjunto de mecanismos que permiten la adquisición, producción, almacenamiento, tratamiento, comunicación, registro y presentación de informaciones, en forma de voz, imágenes y datos contenidos en señales de naturaleza acústica, óptica o electromagnética. Las TIC incluyen la electrónica como tecnología base que soporta el desarrollo de las telecomunicaciones, la informática y el audiovisual.

El profesor Julio Cabero (1996), citado Valcázar, ha sintetizado las características distintivas de las nuevas tecnologías en los siguientes rasgos: inmaterialidad, interactividad, instantaneidad, innovación, elevados parámetros de calidad de imagen y sonido, digitalización, influencia más sobre los procesos que sobre los productos, automatización, interconexión y diversidad.

Inmaterialidad. Las redes informáticas eliminan la necesidad, que durante muchos años se tenía, de coincidir en el espacio y tiempo para la participación en actividades.

Las TIC, en concreto la posibilidad de digitalización, convierten a la información, tradicionalmente sujeta a un medio físico, esto es a todas las condiciones que el medio imponía, en inmaterial. Mediante la digitalización es posible almacenar grandes cantidades de información, en dispositivos físicos de pequeño tamaño (discos, CD, etc.). A su vez los usuarios pueden acceder a información ubicada en dispositivos electrónicos lejanos, que se transmite utilizando las redes de comunicación, de una forma transparente e inmaterial.

Esta característica, ha venido a definir lo que se ha denominado como "realidad virtual".

Podemos transmitir la información instantáneamente a lugares muy alejados físicamente, mediante las denominadas.
Se han acuñado términos como ciberespacio, para definir el espacio virtual, no real, en el que se sitúa la información, al no asumir las características físicas del objeto utilizado para su almacenamiento, adquiriendo ese grado de inmediatez e inmaterialidad.

- Las aplicaciones o programas multimedia han sido desarrollados como un interface amigable y sencillo de comunicación, para facilitar el acceso a las TIC de todos los usuarios. Las características más importantes de estos entornos son:

- Interactividad: Es posiblemente la característica más significativa. Mientras que las tecnologías más clásicas (TV, radio) permiten una interacción unidireccional, del medio al usuario, esto es de un emisor a una masa de espectadores pasivos, el uso del ordenador interconectado mediante las redes digitales de comunicación, proporciona una comunicación bidireccional (sincrónica y asincrónica), persona a persona y persona a grupo.

- Información multimedia. diferentes medios (texto, imagen, sonido, animaciones, etc

Esto en lo que algunos plantean como la Sociedad del Conocimiento y del Aprendizaje, por cuanto hay un afán desmesurado por la proliferación de herramientas para generar, almacenar, transmitir y acceder a la información y la constatación de que la "materia prima" más preciada en este momento es la propia información, la generación de conocimiento y la capacidad para seguir aprendiendo a lo largo de toda la vida, han llevado a conceptos como Sociedad del Conocimiento.

La flexibilidad parece resumir la nueva forma de entender la formación. Flexibilidad de tiempos, de espacios, de conocimientos, de tareas, de relaciones, de trabajo, etc. Flexibilidad entendida como un 
valor que el trabajador debe poseer para poder seguir aprendiendo. Flexibilidad para acomodarse a nuevas situaciones y contextos laborales, para desplazarse sólo o con su familia a otro país o continente; flexibilidad para aprender nuevas habilidades en el lugar donde se encuentre, en el trabajo, en casa o en un hotel; flexibilidad para coordinarse con trabajadores de otras partes del mundo que participan en el proceso de producción dentro de su propia empresa (Marcelo, 2001). Citado por Valcázar (2008).

Lógicamente estas determinantes de la nueva sociedad trae consigo muchas limitaciones, entre las cuales se podrían enumerar:

\section{Limitaciones}

- proliferación de grandes medios de la información

- Afectación de las relaciones humanas

- Concentración de la información en grandes agencias. Se calcula que alrededor del $90 \%$ de las noticias extranjeras que aparecen en los medios de comunicación proceden de las cuatro grandes agencias occidentales, con lo que el punto de vista y la selección de las noticias, en consecuencia, es occidental

- La mundialización de estilos y culturas. Para algunos la perdida de identidad.

- La libertad heterolibertad...libertad aparente.

- Manipulación de la información. UNESCO titulado Un solo mundo, voces múltiples, denuncia el control ejercido por las transnacionales sobre los medios de comunicación y propone sustituir la comunicación en sentido único por un intercambio de mensajes en todas direcciones y completar la comunicación vertical con la horizontal

- La existencia de desigualdades ante las nuevas tecnologías ha sido denunciada desde distintos ámbitos. $47 \%$ de los hogares de americanos blancos tienen ordenador en casa, y el $26,7 \%$ tiene acceso a Internet. En Europa $30.8 \%$, tienen ordenador y conectados a Internet es de $8.3 \%$.

\section{La educación ante el desarrollo de las TIC}

Así como existen problemas y peligros en la educación para la utilización de las TIC, existen también posibilidades, las cuales han sido ya previstas por autores como McClaren en los análisis de los peligros y potencialidades de los mas-media $\mathrm{y}$ las tics.

\section{Valcázar por ejemplo propone el soft- ware educativo como posibilidad.}

El uso del ordenador desde un punto de vista didáctico pasa por el uso del denominado software educativo, estos materiales que suponen utilizar el ordenador con una finalidad didáctica. La funcionalidad del software educativo vendrá determinada por las características y el uso que se haga del mismo, de su adecuación al contexto y la organización de las actividades de enseñanza. Sin embargo, se pueden señalar algunas funciones que serían propias de este medio (Marqués, 1996) citado por Valcázar.

- Función informativa: se presenta una información estructurada de la realidad.

- Función instructiva: orientan el aprendizaje de los estudiantes, facilitando el logro de determinados objetivos educativos.

- Función motivadora: los estudiantes se sienten atraídos por este tipo de material, ya que los programas suelen incluir elementos para captar la atención de los alumnos y mantener su interés (actividad, refuerzos, presentación atractiva...)

- Función evaluadora: la mayoría de los programas ofrece constante feedback 
sobre las actuaciones de los alumnos, corrigiendo de forma inmediata los posibles errores de aprendizaje, presentando ayudas adicionales cuando se necesitan, etc. Se puede decir que ofrecen una evaluación continua y en algunos casos también una evaluación final o explícita, cuando el programa presenta informes sobre la actuación del alumno (número de errores cometidos, tiempo invertido en el aprendizaje, etc.).

- Función investigadora: muchos programas ofrecen interesantes entornos donde investigar: buscar informaciones, relacionar conocimientos, obtener conclusiones, compartir y difundir la información, etc.

- Función expresiva: los estudiantes se pueden expresar y comunicar a través del ordenador, generando materiales con determinadas herramientas, utilizando lenguajes de programación, etc.

- Función metalingüística: los estudiantes pueden aprender los lenguajes propios de la informática.

- Función lúdica: el trabajo con ordenadores tiene para los alumnos en muchos casos connotaciones lúdicas pero además los programas suelen incluir determinados elementos lúdicos.

- Función innovadora: supone utilizar una tecnología recientemente incorporada a los centros educativos que permite hacer actividades muy diversas a la vez que genera diferentes roles tanto en los profesores como en los alumnos e introduce nuevos elementos organizativos en la clase.

- Función creativa: la creatividad se relaciona con el desarrollo de los sentidos (capacidades de observación, percepción y sensibilidad), con el fomento de la iniciativa personal (espontaneidad, autonomía, curiosidad) y el despliegue de la imaginación (desarrollando la fantasía, la intuición, la asociación). Los programas informáticos pueden incidir, pues, en el desarrollo de la creatividad, ya que permiten desarrollar las capacidades indicadas

\section{Los proyectos telemáticos son otra posibilidad de las tics en la educación}

Si a las posibilidades de los materiales didácticos digitales, añadimos la posibilidad de conectar entre sí un determinado material con otros textos o documentos ubicados en distintos ordenadores mediante una red telemática entonces la potencialidad educativa del material se incrementa de forma notoria.

Entendemos la telemática como la comunicación entre ordenadores a distancia, por tanto, la posibilidad de que personas situadas en diferentes contextos sociales puedan intercambiar información. Las comunicaciones a través de las autopistas de la información, básicamente Internet, adquieren una nueva dimensión al suprimir las distancias físicas. Las herramientas como el correo electrónico, las teleconferencias, la transferencia de ficheros, las páginas web, los foros de debate, etc. ofrecen tal cantidad de información y posibilidades de interactividad que el gran problema actual radica en saber localizar y seleccionar la información realmente significativa.

De forma esquemática, podemos decir que la telemática aporta a la educación algunas posibilidades como:

- Eliminar las barreras espacio-temporales entre el profesor y el estudiante.

- Favorecer tanto el aprendizaje colaborativo como el autoaprendizaje.

- Potenciar el aprendizaje a lo largo de toda la vida.

- Interactividad entre los participantes en la educación (profesores, padres, alumnos).

- Ayudar de forma especializada a los 
sujetos con necesidades educativas especiales.

- Favorecer una aproximación de la escuela a la sociedad (conocer el mundo real).

- Combatir el aislamiento de muchas escuelas.

- Facilitar la formación del profesorado.

- Desarrollar tareas de comunicación permitiendo el intercambio de información (escribir para un público real).

- Posibilitar el trabajo sobre temas sociales, permitiendo la aproximación a muy distintas realidades.

- Favorecer la interdisciplinariedad y la globalización.

- Facilitar el acceso a información (bases de datos, foros temáticos, páginas web...).

- Favorecer un acercamiento a la información desde una perspectiva constructivista: los proyectos telemáticos se configuran como pequeñas investigaciones, se basan en la actividad de los estudiantes, fomenta un aprendizaje cooperativo, ...
Definir nuevos roles para profesores y alumnos, de modo que los alumnos asuman una mayor actividad e implicación en el aprendizaje y el educador asuma el papel de ayudar en el proceso de transformar las informaciones en conocimiento, actuando como animador, coordinador y motivador del aprendizaje.

De todas formas, frente a la sociedad de la información y el flujo de capitales de todo tipo, se presentan opciones de gran trascendencia como las definidas por Sen en el marco de unas nuevas perspectivas de desarrollo hacia la expansión de las libertades humanas. La tarea de la educación estaría justamente en buscar mecanismos que permitan dirigir la utilización de las TICS hacia este tipo de perspectivas. 


\section{Bibliografía}

Bauman, Z. (1999). Sección de obras de sociología. Fondo de cultura económica. Brasil: Primera edición en inglés 1998. Primera edición en español 1999.

Barbero, J. M. 2003. Modernidad posmodernidad y modernidades. Discurso sobre la crisis y la diferencia. www.javeriana.edu. $\mathrm{co} /$ pensar/dissens $16 \mathrm{html}$.

Durand, G. (1968). La imaginación simbólica, Buenos Aires, Argentina: Amorrortu.

Feixa, C. (2000). Generación a- la juventud en la era digital. En: revista nómadas No. 13. (pp. 76-91). Bogotá: Universidad central. DIUC, octubre de 2000

Husserl, E. (1997). Filosofía Contemporánea. Ideas Relativas a una Fenomenología Pura y a una Filosofía Fenomenológica. Libro segundo. Investigaciones Fenomenológicas sobre Institución. México,D.c.: Universidad Nacional Autónoma de México.

Lyotard J. F. 1998). La condición posmoderna. Informe sobre el saber. Traducción de Mariano Antolín Rato. Sexta edición. Madrid: Catedra. Teorema.

Mclaren M. (1997). Pedagogía crítica y cultura depredadora. Política de oposición en la era posmoderna. Barcelona: Paidós

Pintos, J. M. 2003. La nueva plausibilidad: La observación de segundo orden en Niklas Luhmann. Santiago de Compostela; Marzo 1994.http://www.imaginaire.com/
Ponty, M. M. (2000). Fenomenología de la percepción. Quinta ed. Barcelona: ediciones península. Trad. Jem Cabarnes.

Sen, A. (2003). Globalización. Consecuencias Humanas. Juicios sobre Globalización.

Sergio, M. (1999) Um Corte Epistemológico. Da Educao Física a Motricidade Humana. Epistemología y Sociedade. Instituto Piaget. Lisboa.

Silva, A. (2.000). Imaginarios Urbanos. $4^{\mathrm{a}}$ edición, Bogotá, Colombia: Tercer mundo Editores.

Sitglitz, E. J. (2001). Developmen thinking at the millennium. Annual work Bank conference on development. Economics 2000. Edited by Boris Pleskovic and Nicholas Stern. The Work bank Washingtong D.C. First Priting July 2001, pp.13-38.

Suárez, R. 1991. La educación. México: trillas 1991.

Valcázar, M. Ana G. (2008). Educación y tecnología. Universidad de Salamanca. www. educacionytecnologias 